\title{
AN EXPLICIT CHARACTERIZATION OF ADMISSIBLE LINEAR ESTIMATORS OF FIXED AND RANDOM EFFECTS IN BALANCED RANDOM MODELS
}

\author{
BY \\ EWA SYNÓWKA - BEJENKA (ZIELONA GóRA) AND \\ STEFAN ZONTEK (ZIELONA GÓRA)
}

\begin{abstract}
A necessary and sufficient conditions for a linear estimator of a linear function of fixed and random effects in a balanced random model to be admissible are given. The formulae for admissible estimators depend on certain coefficients from the interval $[0,1]$, as in well-known results for other models (see e.g. Cohen [3]).
\end{abstract}

2020 Mathematics Subject Classification: Primary 62F10, 62C15; Secondary 62J10.

Key words and phrases: balanced random models, linear estimation, linear prediction, admissibility among an affine set, locally best estimator.

\section{INTRODUCTION}

The problem of simultaneous linear estimation of fixed and random effects was initiated by Henderson [7], who was interested in estimating genetic parameters in animal breeding. Further results on unbiased estimation of both kinds of effects were obtained, among others, by Goldberger [4], Henderson ([8], [9]), Harville [6], Rao [19], Jiang [10], Liu et al. [16] and Tian [27]. Synówka-Bejenka and Zontek ([25], [26]) have dealt with admissibility of linear estimators of fixed and random effects in some linear models. To get a characterization, they have shown that the problem of admissibility for a linear function of fixed and random effects is equivalent to the problem of admissibility for a linear function of the expected value only, in another properly defined linear model (called the dual model; see Section 3 for more details). This reduction allows the use of the results concerning admissibility of linear estimators of linear functions of expected value in a general linear model. Using a duality, Synówka-Bejenka and Zontek [25] obtained a characterization of linear admissible estimators of a linear function of fixed and random effects in the multi-way balanced nested classification random model and in the multi-way balanced crossed classification random model (see also Shiqing et al. [21]). They 
have proved, using a stepwise procedure elaborated by LaMotte [13], that limits of ULBEs are admissible.

The problem of admissibility of linear estimators has received considerable attention in the literature. Despite this, explicit characterizations have been obtained only for special cases. Basing on algebraic properties of matrices Cohen [3] has described all admissible linear estimators of the mean vector in a Gauss-Markov model with identity covariance matrix. Further generalizations have been given, among others, by Rao [18], Mathew et al. [17], Stępniak [22], Zontek [28], Klonecki and Zontek [12], Baksalary and Markiewicz [1], Baksalary et al. [2], Groß and Markiewicz [5] and Stępniak [24]. The problem of characterizing admissible linear estimators of a linear function of expected value was also considered in terms of connection between the closure of the set of unique locally best estimators (ULBE) and the set of admissible linear estimators. This approach was applied, among others, by Stępniak [23], Zontek [29] and LaMotte [15]. LaMotte has shown that every admisible linear estimator is the limit of linear estimators that are uniquely best at points in the minimal closed convex cone containing the original parameter set. On the other hand, he gave an example of a model in which a limit of ULBEs may not be admissible (see LaMotte [14]).

Basing on LaMotte's results [13] Synówka-Bejenka and Zontek [26] have proved that for linear models with finitely generated parameter space every limit of a sequence of ULBEs is admissible. To prove that, they applied a stepwise procedure of LaMotte [13]. Thus they showed that for such models the class of all admissible linear estimators consists of all ULBEs and their limits. For example, they described this class in a model dual to a random linear model for spatially located sensors measuring intensity of a source of signals at discrete instants of time. A special case of this model is the so called one-way balanced random model.

In this paper we note that for any model dual to a balanced random model the parameter space is finitely generated. In such models it is enough to present formulas for ULBEs in the form for which their limits can be characterized. We will use this approach to give explicit formulas for linear admissible estimators of a linear function of fixed and random effects in a balanced random model.

Throughout this paper, $\mathcal{M}_{m \times q}$ denotes the space of $m \times q$ real matrices. The symbols $A^{\prime}$ and $\mathcal{R}(A)$ stand for the transpose and column space of $A \in \mathcal{M}_{m \times q}$, respectively. For $A_{1} \in \mathcal{M}_{m_{1} \times q_{1}}$ and $A_{2} \in \mathcal{M}_{m_{2} \times q_{2}}$ the symbols $A_{1} \otimes A_{2}$ and $\operatorname{diag}\left(A_{1}, A_{2}\right)$ denote the Kronecker product and the matrix whose diagonal consists of $A_{1}$ and $A_{2}$, respectively. The minimal closed convex cone containing a set $A \subset \mathcal{M}_{m \times m} \times \mathcal{M}_{m \times m}$ will be denoted by $[A]$. We write $I_{m}$ and $\mathbf{1}_{m}$ for the $m \times m$ identity matrix and the $m$-vector of ones, respectively.

\section{BALANCED RANDOM MODELS}

Let $Y_{i_{1}, \ldots, i_{r}}$, where $i_{j}=1, \ldots, n_{j}$ for $j=1, \ldots, r$, be a random variable having the following structure (compare Khuri et al. [11]): 


$$
Y_{i_{1}, i_{2}, \ldots, i_{r}}=\beta+u_{1 \psi_{1}}+u_{2 \psi_{2}}+\cdots+u_{k \psi_{k}}+e_{i_{1}, i_{2}, \ldots, i_{r}}
$$

where $\beta$ is an unknown parameter (fixed effect), the term $u_{i \psi_{i}}$ denotes the $i$ th random effect and the symbol $\psi_{i}$ will be identified with the set of subscripts for the $i$ th effect. The last term in (2.1) denotes a random experimental error. We assume that $u_{1 \psi_{1}}, \ldots, u_{k \psi_{k}}$ and $e_{i_{1}, \ldots, i_{r}}$ are uncorrelated random variables with zero mean and variances $\sigma_{1}^{2}, \ldots, \sigma_{k}^{2}$ and $\sigma_{k+1}^{2}$, respectively. To simplify the notation let $\psi_{0}=$ $\emptyset$ and $\psi_{k+1}=\left\{i_{1}, \ldots, i_{r}\right\}$. Arranging the $Y_{i_{1}, \ldots, i_{r}}$ in lexicographic order into an $n$-vector $Y$, where $n=\prod_{j=1}^{r} n_{j}$, we get

$$
Y=Z_{0} \beta+Z_{1} u_{1}+\cdots+Z_{k} u_{k}+Z_{k+1} e,
$$

where the matrix $Z_{i}$ is the Kronecker product

$$
Z_{i}=\bigotimes_{j=1}^{r} N_{i j}, \quad i=0,1, \ldots, k+1
$$

where

$$
N_{i j}= \begin{cases}I_{n_{j}} & \text { when } i_{j} \in \psi_{i} \\ \mathbf{1}_{n_{j}} & \text { otherwise. }\end{cases}
$$

Of course $Z_{0}=\mathbf{1}_{n}$ and $Z_{k+1}=I_{n}$. Clearly,

$$
\mathrm{E}(Y)=Z_{0} \beta \quad \text { and } \quad \operatorname{cov}(Y)=\sum_{i=1}^{k} \sigma_{i}^{2} Z_{i} Z_{i}^{\prime}+\sigma_{k+1}^{2} I_{n}
$$

This will be schematically written as

$$
Y \sim\left(Z_{0} \beta, \sum_{i=1}^{k+1} \sigma_{i}^{2} Z_{i} Z_{i}^{\prime}\right)
$$

This structure covers the well-known examples of balanced random models, e.g. the multi-way nested classification model or the multi-way crossed classification model (with or without interactions). Such models are widely used in various areas of scientific research. Many examples of their applications are described by Sahai and Ojeda [20].

To obtain a characterization of admissible linear estimators of fixed and random effects in the model (2.4), we use some properties of balanced models. Directly from (2.3) we have

$$
\left(Z_{i} Z_{i}^{\prime}\right)^{2}=p_{i} Z_{i} Z_{i}^{\prime}, \quad i=0,1, \ldots, k+1
$$

where

$$
p_{i}= \begin{cases}\prod_{i_{l} \notin \psi_{i}} n_{l} & \text { when } \psi_{i} \neq\left\{i_{1}, \ldots, i_{r}\right\} \\ 1 & \text { when } \psi_{i}=\left\{i_{1}, \ldots, i_{r}\right\}\end{cases}
$$


Note that 2.5 implies that $\frac{1}{p_{i}} Z_{i} Z_{i}^{\prime}$ is an orthogonal projector on $\mathcal{R}\left(Z_{i}\right)$. Moreover, an orthogonal and idempotent basis of the space generated by the matrices $Z_{0} Z_{0}^{\prime}, Z_{1} Z_{1}^{\prime}, \ldots, Z_{k+1} Z_{k+1}^{\prime}$ has the form

$$
\left(E_{0}, E_{1}, \ldots, E_{k+1}\right)^{\prime}=\left((P \Delta)^{-1} \otimes I_{n}\right)\left(Z_{0} Z_{0}^{\prime}, Z_{1} Z_{1}^{\prime}, \ldots, Z_{k+1} Z_{k+1}^{\prime}\right)^{\prime},
$$

where

$$
\begin{aligned}
& P=\operatorname{diag}\left\{p_{0}, p_{1}, \ldots, p_{k+1}\right\}, \\
& \Delta=\left(\delta_{i j}\right)= \begin{cases}1 & \text { when } \psi_{j} \subset \psi_{i}, \\
0 & \text { when } \psi_{j} \not \subset \psi_{i} .\end{cases}
\end{aligned}
$$

If $Z_{1} Z_{1}^{\prime}, \ldots, Z_{k} Z_{k}^{\prime}$ are ordered in such a way that

$$
\mathcal{R}\left(\sum_{j=1}^{i} Z_{j} Z_{j}^{\prime}\right) \subsetneq \mathcal{R}\left(\sum_{j=1}^{i+1} Z_{j} Z_{j}^{\prime}\right), \quad i=1, \ldots, k-1,
$$

then $\Delta$ is a lower triangular matrix. Putting $\Lambda=\left(\lambda_{i j}\right)=\Delta^{-1}$ we get $E_{i}=$ $\sum_{j=0}^{i} \frac{\lambda_{i j}}{p_{j}} Z_{j} Z_{j}^{\prime}$ for $i=0,1, \ldots, k+1$.

From (2.6) one can check the following relationships between the matrices $Z_{i} Z_{i}^{\prime}$ and $E_{i}, i=0,1, \ldots, k+1$ (compare Khuri et al. [11]):

(i) $Z_{i} Z_{i}^{\prime}=p_{i} \sum_{j=0}^{i} \delta_{i j} E_{j}, i=0,1, \ldots, k+1$,

(ii) $E_{j} Z_{i} Z_{i}^{\prime}=p_{i} \delta_{i j} E_{j}, i, j=0,1, \ldots, k+1$.

To obtain a characterization of linear admissible estimators of a linear function of fixed and random effects in a balanced random model we use our earlier results on some duality rule. Section 3 describes background for balanced random models.

\section{BACKGROUND}

Let $Y$ be a random $n$-vector for which the expected value $\mathrm{E} Y$ and the covariance $\operatorname{cov}(Y)$ are given by 2.4. We are interested in admissible estimation of

$$
\theta=\left[\left(K^{\prime} Z_{0} \beta\right)^{\prime},\left(Q_{1}^{\prime} Z_{1} u_{1}\right)^{\prime}, \ldots,\left(Q_{k}^{\prime} Z_{k} u_{k}\right)^{\prime}\right]^{\prime}
$$

in the class of linear estimators

$$
L^{\prime} Y=\left(L_{0}, L_{1}, \ldots, L_{k}\right)^{\prime} Y,
$$

where $K, L_{0} \in \mathcal{M}_{n \times t_{0}} ; Q_{1}, L_{1} \in \mathcal{M}_{n \times t_{1}} ; \ldots ; Q_{k}, L_{k} \in \mathcal{M}_{n \times t_{k}}$. To compare estimators we use the ordinary quadratic risk function

$$
\mathrm{E}\left[\left(L^{\prime} Y-\theta\right)^{\prime}\left(L^{\prime} Y-\theta\right)\right] .
$$


Synówka-Bejenka and Zontek [25] have shown that the quadratic risk function of the estimator $L^{\prime} Y$ of $\theta$ in the model (2.4) is equal to the quadratic risk function of the linear estimator $\boldsymbol{L}^{\prime} \boldsymbol{Y}$ of $\boldsymbol{K}^{\prime} \boldsymbol{X} \beta$ in the dual model, which is defined by

$$
\boldsymbol{Y}=\left[Y^{\prime},\left(Z_{1} u_{1}\right)^{\prime}, \ldots,\left(Z_{k} u_{k}\right)^{\prime}\right]^{\prime} \sim\left(\boldsymbol{X} \beta, \sum_{i=1}^{k+1} \sigma_{i}^{2} \boldsymbol{V}_{i}\right)
$$

where

$$
\begin{aligned}
\boldsymbol{X} & =\left(Z_{0}^{\prime}, \mathbf{0}, \ldots, \mathbf{0}\right)^{\prime}, \\
\boldsymbol{V}_{i} & =\left(v_{1}+v_{i+1}\right)\left(v_{1}+v_{i+1}\right)^{\prime} \otimes Z_{i} Z_{i}^{\prime}, \quad i=1, \ldots, k, \\
\boldsymbol{V}_{k+1} & =v_{1} v_{1}^{\prime} \otimes I_{n},
\end{aligned}
$$

while $v_{i}$ is the $i$ th versor in $\mathbb{R}^{k+1}$. This means that a linear estimator $L^{\prime} Y$ of $\theta$ is admissible in the model (2.4) if and only if the corresponding estimator

$$
\boldsymbol{L}^{\prime} \boldsymbol{Y}=\left[\begin{array}{cccc}
L_{0} & L_{1} & \cdots & L_{k} \\
\mathbf{0} & -Q_{1} & \cdots & \mathbf{0} \\
\vdots & \vdots & \ddots & \vdots \\
\mathbf{0} & \mathbf{0} & \cdots & -Q_{k}
\end{array}\right]^{\prime}\left[\begin{array}{c}
Y \\
Z_{1} u_{1} \\
\vdots \\
Z_{k} u_{k}
\end{array}\right]
$$

of

$$
\boldsymbol{K}^{\prime} \mathrm{E} \boldsymbol{Y}=\left[\begin{array}{cccc}
K & \mathbf{0} & \cdots & \mathbf{0} \\
\mathbf{0} & \mathbf{0} & \cdots & \mathbf{0} \\
\vdots & \vdots & \ddots & \vdots \\
\mathbf{0} & \mathbf{0} & \cdots & \mathbf{0}
\end{array}\right]^{\prime}\left[\begin{array}{c}
Z_{0} \beta \\
\mathbf{0} \\
\vdots \\
\mathbf{0}
\end{array}\right]
$$

is admissible in the model (3.3).

Note that the class of linear estimators of $\boldsymbol{K}^{\prime} \mathrm{E} \boldsymbol{Y}$ considered in the model 3.3 is restricted to the set

$$
\mathcal{E}_{0}=\left\{\boldsymbol{L}^{\prime} \boldsymbol{Y}: \boldsymbol{L} \in \mathcal{L}_{0}\right\}
$$

where $\mathcal{L}_{0}$ is the affine set given by

$$
\mathcal{L}_{0}=\left\{\boldsymbol{L}_{0}+\boldsymbol{\Pi}_{0} \boldsymbol{M}: \boldsymbol{M} \in \mathcal{M}_{(k+1) n \times\left(t_{0}+\cdots+t_{k}\right)}\right\},
$$

while $\boldsymbol{L}_{0}=\operatorname{diag}\left(\mathbf{0},-Q_{1}, \ldots,-Q_{k}\right)$ and $\boldsymbol{\Pi}_{0}=v_{1} v_{1}^{\prime} \otimes I_{n}$. Following LaMotte [13], consider the set

$$
\mathcal{T}=\left\{\left(\operatorname{cov}(\boldsymbol{Y}), \operatorname{E} \boldsymbol{Y}(\mathrm{E} \boldsymbol{Y})^{\prime}\right): \beta \in \mathbb{R}, \sigma_{1}^{2} \geqslant 0, \ldots, \sigma_{k+1}^{2} \geqslant 0\right\}
$$

as a new space of parameters and a point $\left(W_{1}, W_{2}\right) \in[\mathcal{T}]$ as an argument of an extended quadratic risk function of $\boldsymbol{L}^{\prime} \boldsymbol{Y}$, i.e.,

$$
\mathrm{R}\left(\boldsymbol{L}^{\prime} \boldsymbol{Y} ;\left(W_{1}, W_{2}\right)\right)=\operatorname{tr}\left[\boldsymbol{L}^{\prime} W_{1} \boldsymbol{L}+(\boldsymbol{L}-\boldsymbol{K})^{\prime} W_{2}(\boldsymbol{L}-\boldsymbol{K})\right] .
$$


Recall that an estimator $\boldsymbol{L}^{\prime} \boldsymbol{Y}$ with $\boldsymbol{L} \in \mathcal{L}$, where $\mathcal{L}$ is an affine subset of $\mathcal{L}_{0}$, is called locally best among $\mathcal{L}$ at a point $\left(W_{1}, W_{2}\right) \in[\mathcal{T}]$ if

$$
\mathrm{R}\left(\boldsymbol{L}^{\prime} \boldsymbol{Y} ;\left(W_{1}, W_{2}\right)\right) \leqslant \mathrm{R}\left(\boldsymbol{N}^{\prime} \boldsymbol{Y} ;\left(W_{1}, W_{2}\right)\right)
$$

for every $N \in \mathcal{L}$. LaMotte [13] has shown that an estimator $\boldsymbol{L}^{\prime} \boldsymbol{Y}$ is locally best among $\mathcal{L}$ at $\left(W_{1}, W_{2}\right) \in[\mathcal{T}]$ iff

$$
\boldsymbol{\Pi}^{\prime}\left(W_{1}+W_{2}\right) \boldsymbol{L}=\boldsymbol{\Pi}^{\prime} W_{2} \boldsymbol{K},
$$

where $\Pi$ is a $((k+1) n \times(k+1) n)$-matrix such that

$$
\mathcal{L}=\left\{\boldsymbol{L}+\boldsymbol{\Pi} \boldsymbol{M}: \boldsymbol{M} \in \mathcal{M}_{(k+1) n \times\left(t_{0}+\cdots+t_{k}\right)}\right\} .
$$

Note that the parameter space given by $(3.5)$ corresponding to the dual model (3.3) is a finitely generated closed convex cone, i.e.,

$$
\mathcal{T}=[\mathcal{T}]=\left\{\sum_{i=0}^{k+1} t_{i}\left(W_{1 i}, W_{2 i}\right): t_{0} \geqslant 0, \ldots, t_{k+1} \geqslant 0\right\}
$$

where

$$
\begin{aligned}
\left(W_{10}, W_{20}\right) & =\left(\mathbf{0}, \boldsymbol{X} \boldsymbol{X}^{\prime}\right), \\
\left(W_{1 i}, W_{2 i}\right) & =\left(\boldsymbol{V}_{i}, \mathbf{0}\right), \quad i=1, \ldots, k+1 .
\end{aligned}
$$

To avoid trivialities we also assume that

$$
\mathcal{R}\left(\sum_{i=0}^{k+1} \boldsymbol{\Pi}^{\prime}\left(W_{1 i}+W_{2 i}\right) \boldsymbol{\Pi}\right)=\mathcal{R}\left(\boldsymbol{\Pi}^{\prime}\right)
$$

and

$$
\boldsymbol{\Pi}^{\prime}\left(W_{1 i}+W_{2 i}\right) \boldsymbol{\Pi} \neq 0 \quad \text { for } i=0, \ldots, k+1 .
$$

In the next section we apply this approach to obtain an explicit characterization of admissible linear estimators of fixed and random effects in the model (2.4).

\section{MAIN RESULT}

To characterize admissible estimators $\boldsymbol{L}^{\prime} \boldsymbol{Y}$ for $\boldsymbol{K}^{\prime} \mathrm{E} \boldsymbol{Y}$ among $\mathcal{L}_{0}$ in the model (3.3) corresponding to 2.4 we prove the following lemma, which gives necessary and sufficient conditions for an estimator $\boldsymbol{L}^{\prime} \boldsymbol{Y}$ to be ULBE at $\left(W_{1}, W_{2}\right) \in \mathcal{T}$.

LEMma 4.1. An estimator $\boldsymbol{L}^{\prime} \boldsymbol{Y}$ is ULBE at $\left(\sum_{i=1}^{k+1} s_{i} \boldsymbol{V}_{i}, s_{0} \boldsymbol{X} \boldsymbol{X}^{\prime}\right)$ in $\mathcal{T}$ among $\mathcal{L}_{0}$ in the model (3.3) corresponding to (2.4) if and only if $s_{0} \geqslant 0, \ldots$, $s_{k} \geqslant 0, s_{k+1}>0$ and

where

$$
\boldsymbol{L}=\left[\begin{array}{cccc}
L_{0} & L_{1} & \cdots & L_{k} \\
\mathbf{0} & -Q_{1} & \cdots & \mathbf{0} \\
\vdots & \vdots & \ddots & \vdots \\
\mathbf{0} & \mathbf{0} & \cdots & -Q_{k}
\end{array}\right]
$$




$$
\begin{aligned}
L_{0} & =\frac{s_{0} p_{0}}{w_{0}} E_{0} K, \\
L_{i} & =\frac{s_{i} p_{i}}{w_{i}}\left(\sum_{j=0}^{i} \delta_{i j} \frac{w_{i}}{w_{j}} E_{j}\right) Q_{i} \quad \text { for } i=1, \ldots, k,
\end{aligned}
$$

while

$$
w_{i}=\sum_{l=i}^{k+1} s_{l} p_{l} \delta_{l i} \quad \text { for } i=0, \ldots, k+1 .
$$

Proof. An estimator $\boldsymbol{L}^{\prime} \boldsymbol{Y}$ is locally best at $\left(\sum_{i=1}^{k+1} s_{i} \boldsymbol{V}_{i}, s_{0} \boldsymbol{X} \boldsymbol{X}^{\prime}\right)$ in $\mathcal{T}$ among $\mathcal{L}_{0}$ iff $s_{j} \geqslant 0$ for $j=0, \ldots, k+1$ and

$$
\boldsymbol{\Pi}_{0}\left(\sum_{i=1}^{k+1} s_{i} \boldsymbol{V}_{i}+s_{0} \boldsymbol{X} \boldsymbol{X}^{\prime}\right) \boldsymbol{L}=s_{0} \boldsymbol{\Pi}_{0} \boldsymbol{X} \boldsymbol{X}^{\prime} \boldsymbol{K} .
$$

In more detail, this equation can be written as

$$
\begin{aligned}
W L_{0} & =s_{0} X X^{\prime} K, \\
W L_{i}-s_{i} Z_{i} Z_{i}^{\prime} Q_{i} & =\mathbf{0} \quad \text { for } i=1, \ldots, k,
\end{aligned}
$$

where $W=\sum_{i=0}^{k+1} s_{i} Z_{i} Z_{i}^{\prime}$. Of course, the above equations have only one solution $L_{0}, \ldots, L_{k}$ iff the matrix $W$ is nonsingular, that is, $s_{k+1}>0$. Moreover, since by (i),

$$
W=\sum_{i=0}^{k+1} s_{i} Z_{i} Z_{i}^{\prime}=\sum_{i=0}^{k+1} s_{i}\left(p_{i} \sum_{j=0}^{i} \delta_{i j} E_{j}\right)=\sum_{i=0}^{k+1} w_{i} E_{i},
$$

and since $E_{i} E_{j}=0$ for $i \neq j$, we see that $W^{-1}=\sum_{i=0}^{k+1} \frac{1}{w_{i}} E_{i}$. Hence and by (ii) we get

$$
\begin{aligned}
L_{i} & =s_{i} W^{-1} Z_{i} Z_{i}^{\prime} Q_{i}=s_{i}\left(\sum_{j=0}^{k+1} \frac{1}{w_{j}} E_{j}\right) Z_{i} Z_{i}^{\prime} Q_{i}=s_{i}\left(\sum_{j=0}^{k+1} \frac{p_{i} \delta_{i j}}{w_{j}} E_{j}\right) Q_{i} \\
& =\frac{s_{i} p_{i}}{w_{i}}\left(\sum_{j=0}^{i} \delta_{i j} \frac{w_{i}}{w_{j}} E_{j}\right) Q_{i} \text {. }
\end{aligned}
$$

Explicit formulas for linear admissible estimators $\boldsymbol{L}^{\prime} \boldsymbol{Y}$ will be given in terms of certain coefficients $a_{i}$. Let

$$
a=\left(a_{0}, a_{1}, \ldots, a_{k+1}\right)^{\prime}=\left(\frac{s_{0} p_{0}}{w_{0}}, \frac{s_{1} p_{1}}{w_{1}}, \ldots, \frac{s_{k+1} p_{k+1}}{w_{k+1}}\right)^{\prime} .
$$

Note that for any fixed $s_{i+1} \geqslant 0, \ldots, s_{k} \geqslant 0$ and $s_{k+1}>0$ the parameter $a_{i}$ runs over $[0,1)$ when $s_{i} \in[0,+\infty)$ for $i=0, \ldots, k$ and $a_{k+1}=1$. Further, taking

$$
w=\Delta^{\prime} P s
$$

where $w=\left(w_{0}, w_{1}, \ldots, w_{k+1}\right)^{\prime}$ and $s=\left(s_{0}, s_{1}, \ldots, s_{k+1}\right)^{\prime}$, we have

$$
a=\left(\operatorname{diag}\left\{w_{0}, w_{1}, \ldots, w_{k+1}\right\}\right)^{-1} P s=\left(\operatorname{diag}\left\{w_{0}, w_{1}, \ldots, w_{k+1}\right\}\right)^{-1} \Lambda^{\prime} w .
$$


In more detail the last equation can be written as

$$
\left\{\begin{array}{lrrr}
a_{0}= & \lambda_{00}+\lambda_{10} \frac{w_{1}}{w_{0}}+\lambda_{20} \frac{w_{2}}{w_{0}}+\ldots+\lambda_{k 0} \frac{w_{k}}{w_{0}}+\lambda_{k+1,0} \frac{w_{k+1}}{w_{0}} \\
a_{1}= & \lambda_{11}+\lambda_{21} \frac{w_{2}}{w_{1}}+\ldots+\lambda_{k 1} \frac{w_{k}}{w_{1}}+\lambda_{k+1,1} \frac{w_{k+1}}{w_{1}} \\
a_{2}= & \lambda_{22}+\ldots+\lambda_{k 2} \frac{w_{k}}{w_{2}}+\lambda_{k+1,2} \frac{w_{k+1}}{w_{2}} \\
\vdots & \ddots & \vdots & \vdots \\
a_{k}= & & \lambda_{k k} & +\lambda_{k+1, k} \frac{w_{k+1}}{w_{k}}, \\
a_{k+1} & & \lambda_{k+1, k+1} .
\end{array}\right.
$$

Note that we can express the above system in the form

$$
\mathbf{0}=\left(\Lambda^{\prime}-\operatorname{diag}\left\{a_{0}, a_{1}, \ldots, a_{k+1}\right\}\right) w=\left(I-\Delta^{\prime} \operatorname{diag}\left\{a_{0}, a_{1}, \ldots, a_{k+1}\right\}\right) w .
$$

Since $\delta_{i i}=1$ for $i=0,1, \ldots, k+1$, we get

$$
\mathbf{0}=A w
$$

where

$$
A=\left[\begin{array}{cccccc}
1-a_{0} & -a_{1} \delta_{10} & -a_{2} \delta_{20} & \cdots & -a_{k} \delta_{k, 0} & -a_{k+1} \delta_{k+1,0} \\
0 & 1-a_{1} & -a_{2} \delta_{21} & \cdots & -a_{k} \delta_{k, 1} & -a_{k+1} \delta_{k+1,1} \\
0 & 0 & 1-a_{2} & \cdots & -a_{k} \delta_{k, 2} & -a_{k+1} \delta_{k+1,2} \\
\vdots & \vdots & \vdots & \ddots & \vdots & \vdots \\
0 & 0 & 0 & \cdots & 1-a_{k} & -a_{k+1} \delta_{k+1, k} \\
0 & 0 & 0 & \cdots & 0 & 1-a_{k+1}
\end{array}\right]
$$

LEMMA 4.2. The system 4.1 has a non-trivial solution

$$
\begin{aligned}
w_{i}= & \frac{w_{k+1}}{1-a_{i}}\left(1+\sum_{l_{1}=i+1}^{k} \frac{a_{l_{1}} \delta_{l_{1} i}}{1-a_{l_{1}}}+\sum_{l_{1}=i+1}^{k} \sum_{l_{2}=l_{1}+1}^{k} \frac{a_{l_{1}} \delta_{l_{1} i}}{1-a_{l_{1}}} \frac{a_{l_{2}} \delta_{l_{2} l_{1}}}{1-a_{l_{2}}}\right. \\
& +\sum_{l_{1}=i+1}^{k} \sum_{l_{2}=l_{1}+1}^{k} \sum_{l_{3}=l_{2}+1}^{k} \frac{a_{l_{1}} \delta_{l_{1} i}}{1-a_{l_{1}}} \frac{a_{l_{2}} \delta_{l_{2} l_{1}}}{1-a_{l_{2}}} \frac{a_{l_{3}} \delta_{l_{3} l_{2}}}{1-a_{l_{3}}}+\cdots \\
& \left.+\sum_{l_{1}=i+1}^{k} \sum_{l_{2}=l_{1}+1}^{k} \ldots \sum_{l_{k-i}=l_{k-i-1}+1}^{k} \frac{a_{l_{1}} \delta_{l_{1} i}}{1-a_{l_{1}}} \frac{a_{l_{2}} \delta_{l_{2} l_{1}}}{1-a_{l_{2}}} \ldots \frac{a_{l_{k-i}} \delta_{l_{k-i} l_{k-i-1}}}{1-a_{l_{k-i}}}\right)
\end{aligned}
$$

for $i=0,1, \ldots, k$.

Proof. Since $0 \leqslant a_{i}<1$ for $i=0,1, \ldots, k$ and $a_{k+1}=1$ we find that $\operatorname{rank}(A)=k+1$. Moreover, the vector $w=\left(w_{0}, w_{1}, \ldots, w_{k+1}\right)^{\prime}$, where $w_{0}, w_{1}$, $\ldots, w_{k}$ are given by (4.2), can be easily checked to satisfy $\mathbf{0}=A w$. 
THEOREM 4.1. For an estimator $\boldsymbol{L}^{\prime} \boldsymbol{Y}$ of $\boldsymbol{K}^{\prime} \boldsymbol{X} \beta$ to be admissible among $\mathcal{L}_{0}$ in the model (3.3) corresponding to (2.4) it is necessary and sufficient that $\boldsymbol{L}$ belongs to the closure of the set

$$
\left\{\left[\begin{array}{cccc}
L_{0}(a) & L_{1}(a) & \cdots & L_{k}(a) \\
\mathbf{0} & -Q_{1} & \cdots & \mathbf{0} \\
\vdots & \vdots & \ddots & \vdots \\
\mathbf{0} & \mathbf{0} & \cdots & -Q_{k}
\end{array}\right]: a=\left(a_{0}, a_{1}, \ldots, a_{k}\right)^{\prime} \in[0,1)^{k+1}\right\}
$$

where

$$
\begin{aligned}
L_{0} & =a_{0} E_{0} K, \\
L_{i} & =a_{i}\left(\sum_{j=0}^{i} \delta_{i j} \frac{w_{i}}{w_{j}} E_{j}\right) Q_{i} \quad \text { for } i=1, \ldots, k,
\end{aligned}
$$

while $w_{i}$ and $w_{j}$ are given by 4.2 .

Proof. Necessity. Let $\boldsymbol{L}$ belong to the set (4.3) with $a_{i} \in[0,1)$ for $i=$ $0,1, \ldots, k$. Using Lemma 4.1 it can be checked that $\boldsymbol{L}^{\prime} \boldsymbol{Y}$ is an ULBE at $W=$ $\left(\sum_{i=1}^{k+1} s_{i} \boldsymbol{V}_{i}, s_{0} \boldsymbol{X} \boldsymbol{X}^{\prime}\right)$ in $\mathcal{T}$ given by

$$
\begin{aligned}
s_{i} & =\frac{a_{i}}{p_{i}} \frac{w_{i}}{w_{k+1}} s_{k+1} \quad \text { for } i=0,1, \ldots, k, \\
s_{k+1} & >0 .
\end{aligned}
$$

Since for any fixed $a_{i+1} \in[0,1), \ldots, a_{k} \in[0,1)$ the value $s_{i}$ runs over $[0,+\infty)$ when $a_{i} \in[0,1)$ for $i=0, \ldots, k$, the set given by $[4.3]$ is the closure of

$\left\{\boldsymbol{M}: \boldsymbol{M}^{\prime} \boldsymbol{Y}\right.$ is an ULBE at a point of $\mathcal{T}$ among $\left.\mathcal{L}_{0}\right\}$.

So the first part of the proof is completed by using a result of LaMotte [15] that each linear estimator of $\boldsymbol{K}^{\prime} \mathrm{E} \boldsymbol{Y}$ admissible among $\mathcal{L}_{0}$ is a limit of members of $\mathcal{L}_{0}$ that are uniquely best among $\mathcal{L}_{0}$ in $\mathcal{T}$.

Sufficiency follows straightforwardly from a result of Synówka-Bejenka and Zontek [26] that for a model with finitely generated parameter space each limit of members of $\mathcal{L}_{0}$ is admissible.

\section{EXAMPLE}

Consider a two-factor study where factors $F_{1}$ and $F_{2}$ have $n_{1}$ and $n_{2}$ levels, respectively, and there are $n_{3}$ replications in each cell. We say that the factors interact if the differences between the mean response at different levels of factor $F_{1}$ tend to vary over the different levels of factor $F_{2}$. The two-way crossed classification random model with interaction, a special case of the model (2.1), is given by

$$
\begin{aligned}
& Y_{i_{1} i_{2} i_{3}}=\beta+u_{1 i_{1}}+u_{2 i_{2}}+u_{3 i_{1} i_{2}}+e_{i_{1} i_{2} i_{3}}, \\
& i_{1}=1, \ldots, n_{1} ; i_{2}=1, \ldots, n_{2} ; i_{3}=1, \ldots, n_{3},
\end{aligned}
$$


where $Y_{i_{1} i_{2} i_{3}}$ is the $i_{3}$ th observation corresponding to the $\left(i_{1}, i_{2}\right)$ th cell, $\beta$ is a constant, the $u_{1 i_{1}}$ are effects due to factor $F_{1}$, the $u_{2 i_{2}}$ are effects due to factor $F_{2}$, and $u_{3 i_{1} i_{2}}$ is the interaction between $u_{1 i_{1}}$ and $u_{2 i_{2}}$. For this model we have

$$
\begin{aligned}
& \psi_{0}=\emptyset, \quad \psi_{1}=\left\{i_{1}\right\}, \quad \psi_{2}=\left\{i_{2}\right\}, \quad \psi_{3}=\left\{i_{1}, i_{2}\right\}, \quad \psi_{4}=\left\{i_{1}, i_{2}, i_{3}\right\}, \\
& Z_{0}=\mathbf{1}_{n_{1}} \otimes \mathbf{1}_{n_{2}} \otimes \mathbf{1}_{n_{3}}, \quad Z_{1}=I_{n_{1}} \otimes \mathbf{1}_{n 2} \otimes \mathbf{1}_{n_{3}}, \quad Z_{2}=\mathbf{1}_{n_{1}} \otimes I_{n 2} \otimes \mathbf{1}_{n_{3}}, \\
& Z_{3}=I_{n_{1}} \otimes I_{n 2} \otimes \mathbf{1}_{n_{3}}, \quad Z_{4}=I_{n_{1}} \otimes I_{n 2} \otimes I_{n_{3}}, \\
& p_{0}=n_{1} n_{2} n_{3}, \quad p_{1}=n_{2} n_{3}, \quad p_{2}=n_{1} n_{3}, \quad p_{3}=n_{3}, \quad p_{4}=1 .
\end{aligned}
$$

Hence

$$
\Delta=\left[\begin{array}{lllll}
1 & 0 & 0 & 0 & 0 \\
1 & 1 & 0 & 0 & 0 \\
1 & 0 & 1 & 0 & 0 \\
1 & 1 & 1 & 1 & 0 \\
1 & 1 & 1 & 1 & 1
\end{array}\right]
$$

while

$$
\begin{aligned}
w_{3}= & \frac{w_{4}}{1-a_{3}}, \\
w_{2}= & \frac{w_{4}}{1-a_{2}}\left(1+\frac{a_{3} \delta_{32}}{1-a_{3}}\right)=\frac{w_{4}}{1-a_{2}}\left(1+\frac{a_{3}}{1-a_{3}}\right)=\frac{w_{4}}{\left(1-a_{2}\right)\left(1-a_{3}\right)}, \\
w_{1}= & \frac{w_{4}}{1-a_{1}}\left(1+\frac{a_{2} \delta_{21}}{1-a_{2}}+\frac{a_{3} \delta_{31}}{1-a_{3}}+\frac{a_{2} \delta_{21} a_{3} \delta_{32}}{\left(1-a_{2}\right)\left(1-a_{3}\right)}\right)=\frac{w_{4}}{\left(1-a_{1}\right)\left(1-a_{3}\right)}, \\
w_{0}= & \frac{w_{4}}{1-a_{0}}\left(1+\frac{a_{1} \delta_{10}}{1-a_{1}}+\frac{a_{2} \delta_{20}}{1-a_{2}}+\frac{a_{3} \delta_{30}}{1-a_{3}}\right. \\
& \quad+\frac{a_{1} \delta_{10} a_{2} \delta_{21}}{\left(1-a_{1}\right)\left(1-a_{2}\right)}+\frac{a_{1} \delta_{10} a_{3} \delta_{31}}{\left(1-a_{1}\right)\left(1-a_{3}\right)}+\frac{a_{2} \delta_{20} a_{3} \delta_{32}}{\left(1-a_{2}\right)\left(1-a_{3}\right)} \\
& \left.\quad+\frac{a_{1} \delta_{10} a_{2} \delta_{21} a_{3} \delta_{32}}{\left(1-a_{1}\right)\left(1-a_{2}\right)\left(1-a_{3}\right)}\right) \\
= & \frac{w_{4}}{1-a_{0}}\left(1+\frac{a_{1}}{1-a_{1}}+\frac{a_{2}}{1-a_{2}}+\frac{a_{3}}{1-a_{3}}\right. \\
& \left.\quad+\frac{a_{1} a_{3}}{\left(1-a_{1}\right)\left(1-a_{3}\right)}+\frac{a_{2} a_{3}}{\left(1-a_{2}\right)\left(1-a_{3}\right)}\right) \\
= & \frac{w_{4}}{1-a_{0}} \frac{1-a_{1} a_{2}}{\left(1-a_{1}\right)\left(1-a_{2}\right)\left(1-a_{3}\right)} .
\end{aligned}
$$

After appropriate transformations we obtain the following explicit formulas for ULBEs:

$$
\begin{aligned}
& L_{0}(a)=a_{0} E_{0} K \\
& L_{1}(a)=a_{1}\left[\delta_{10} \frac{w_{1}}{w_{0}} E_{0}+E_{1}\right] Q_{1}=a_{1}\left[\left(1-a_{0}\right) \frac{1-a_{2}}{1-a_{1} a_{2}} E_{0}+E_{1}\right] Q_{1},
\end{aligned}
$$




$$
\begin{aligned}
L_{2}(a) & =a_{2}\left[\delta_{20} \frac{w_{2}}{w_{0}} E_{0}+\delta_{21} \frac{w_{2}}{w_{1}} E_{1}+E_{2}\right] Q_{2} \\
& =a_{2}\left[\left(1-a_{0}\right) \frac{1-a_{1}}{1-a_{1} a_{2}} E_{0}+E_{2}\right] Q_{2}, \\
L_{3}(a) & =a_{3}\left[\delta_{30} \frac{w_{3}}{w_{0}} E_{0}+\delta_{31} \frac{w_{3}}{w_{1}} E_{1}+\delta_{32} \frac{w_{3}}{w_{2}} E_{2}+E_{3}\right] Q_{3} \\
& =a_{3}\left[\left(1-a_{0}\right) \frac{\left(1-a_{1}\right)\left(1-a_{2}\right)}{1-a_{1} a_{2}} E_{0}+\left(1-a_{1}\right) E_{1}+\left(1-a_{2}\right) E_{2}+E_{3}\right] Q_{3} .
\end{aligned}
$$

For a complete characterization of admissible linear estimators $\boldsymbol{L}^{\prime} \boldsymbol{Y}$ of $\boldsymbol{K}^{\prime} \boldsymbol{X} \beta$, it is enough to give a form of limits of ULBEs. Note that $L_{i}, i=1,2,3$, can be expressed as

$$
\begin{aligned}
L_{1}(a) & =a_{1}\left[\delta_{10} \frac{w_{1}}{w_{0}} E_{0}+E_{1}\right] Q_{1}=a_{1}\left[E_{1}+\left(1-a_{0}\right) A_{1} E_{0}\right] Q_{1}, \\
L_{2}(a) & =a_{2}\left[\delta_{20} \frac{w_{2}}{w_{0}} E_{0}+\delta_{21} \frac{w_{2}}{w_{1}} E_{1}+E_{2}\right] Q_{2}=a_{2}\left[E_{2}+\left(1-a_{0}\right) A_{2} E_{0}\right] Q_{2}, \\
L_{3}(a) & =a_{3}\left[\delta_{30} \frac{w_{3}}{w_{0}} E_{0}+\delta_{31} \frac{w_{3}}{w_{1}} E_{1}+\delta_{32} \frac{w_{3}}{w_{2}} E_{2}+E_{3}\right] Q_{3} \\
& =a_{3}\left[E_{3}+\left(1-a_{0}\right)\left(A_{1}+A_{2}-1\right) E_{0}+\left(1-a_{1}\right) E_{1}+\left(1-a_{2}\right) E_{2}\right] Q_{3},
\end{aligned}
$$

where

$$
A_{1}=\frac{\frac{1}{1-a_{1}}}{\frac{1}{1-a_{1}}+\frac{1}{1-a_{2}}-1}, \quad A_{2}=\frac{\frac{1}{1-a_{2}}}{\frac{1}{1-a_{1}}+\frac{1}{1-a_{2}}-1} \quad \text { for } a_{1}, a_{2} \in[0,1) .
$$

Of course for $A_{1}$ and $A_{2}$ defined by (5.2) we have $1<A_{1}+A_{2} \leqslant 2$. Since $\frac{1}{1-a_{1}}+\frac{1}{1-a_{2}} \rightarrow+\infty$ as $a_{1} \rightarrow 1$ or $a_{2} \rightarrow 1$, we have $A_{1}+A_{2} \rightarrow 1$ and $A_{i} \rightarrow 0$ for $i=1,2$. So each linear estimator of $\boldsymbol{K}^{\prime} \mathrm{E} \boldsymbol{Y}$ admissible among $\mathcal{L}_{0}$ is a limit of members of $\mathcal{L}_{0}$ that are uniquely best among $\mathcal{L}_{0}$ in $\mathcal{T}$.

\section{SIMULATIONS}

In simulation studies we considered the following model:

$$
Y_{i_{1} i_{2}}=\beta+u_{1 i_{1}}+e_{i_{1} i_{2}}, \quad i_{1}=1, \ldots, n_{1}, i_{2}=1, \ldots, n_{2} .
$$

For this model we have

$$
\begin{aligned}
& \psi_{0}=\emptyset, \quad \psi_{1}=\left\{i_{1}\right\}, \quad \psi_{2}=\left\{i_{1}, i_{2}\right\} \\
& Z_{0}=1_{n_{1}} \otimes 1_{n_{2}}, \quad Z_{1}=I_{n_{1}} \otimes 1_{n_{2}}, \quad Z_{2}=I_{n_{1}} \otimes I_{n_{2}}, \\
& p_{0}=n_{1} n_{2}, \quad p_{1}=n_{2}, \quad p_{2}=1 .
\end{aligned}
$$


We choose $K=\frac{1}{p_{0}} Z_{0}$ and $Q_{1}=\frac{1}{p_{1}} Z_{1}$, so that the estimated parameter vector $\theta$ is

$$
\theta=\left(\beta, u_{11}, u_{12}, \ldots, u_{1 n_{1}}\right)^{\prime} .
$$

An admissible estimator of $\theta$ takes the form

$$
L^{\prime} Y=\left(a_{0} E_{0} K, a_{1}\left[E_{1}+\left(1-a_{0}\right) E_{0}\right] Q_{1}\right)^{\prime} Y,
$$

where $a_{0}, a_{1} \in[0,1]$, while $E_{0}=\frac{1}{p_{0}} Z_{0} Z_{0}^{\prime}$ and $E_{1}=\frac{1}{p_{1}} Z_{1} Z_{1}^{\prime}-E_{0}$.

If we decide to compare estimators using the quadratic risk function, then from the theoretical point of view each admissible estimator is a good choice. But admissibility does not guarantee good effects of estimation from the practical point of view. For example, the estimator (6.2) with $a_{0}=a_{1}=0$ is perfect as an estimator of $\theta$ but only for $\mu=0$ and $\sigma_{1}^{2}=0$. When $\mu \neq 0$ and $\sigma_{1}^{2} \neq 0$, it cannot be recommended. Simulations presented below suggest that a satisfactory result is obtained for $a_{0}$ very close to (or equal to) 1 and for $a_{1}$ close to 1 .

For chosen $a_{0}, a_{1} \in[0,1]$ and a fixed number $N$ of generated data denote by

$$
\left(\hat{\beta}_{j}, \hat{u}_{11 j}, \hat{u}_{12 j}, \ldots, \hat{u}_{1 n_{1 j}}\right)^{\prime}, \quad j=1, \ldots, N,
$$

values of estimators of $\theta$.

As a result of simulations, in the tables below we present the means of estimates (the first row for $a_{1}$ given in the first column), that is,

$$
\bar{\beta}=\frac{1}{N} \sum_{j=1}^{N} \hat{\beta}_{j}, \quad \bar{u}_{1 i_{1}}=\frac{1}{N} \sum_{j=1}^{N} \hat{u}_{1 i_{1} j}, \quad i_{1}=1, \ldots, n_{1},
$$

and the average square of the difference between the estimated values and corresponding estimates (the second row for given $a_{1}$, in brackets), i.e.

$$
\frac{1}{N} \sum_{j=1}^{N}\left(\hat{\beta}_{j}-\beta\right)^{2}, \quad \frac{1}{N} \sum_{j=1}^{N}\left(\hat{u}_{1 i_{1} j}-u_{1 i_{1} j}\right)^{2}, \quad i_{1}=1, \ldots, n_{1} .
$$

In simulation we used the following parameters:

$n_{1}=4, \quad n_{2}=10, \quad \beta=10, \quad u_{1 i_{1}} \sim N(0,1), \quad e_{i_{1} i_{2}} \sim N(0,1)$ and $N=10000$.

TABLE 1. Simulation results for $a_{0}=0.90$ and selected $a_{1}$.

\begin{tabular}{cccccc}
\hline 0.85 & 9.015 & 0.848 & 0.848 & 0.854 & 0.856 \\
& $(1.782)$ & $(1.646)$ & $(1.645)$ & $(1.647)$ & $(1.637)$ \\
0.90 & 9.015 & 0.898 & 0.898 & 0.904 & 0.907 \\
& $(1.782)$ & $(1.692)$ & $(1.690)$ & $(1.694)$ & $(1.686)$ \\
0.95 & 9.015 & 0.948 & 0.947 & 0.954 & 0.957 \\
& $(1.782)$ & $(1.759)$ & $(1.755)$ & $(1.762)$ & $(1.755)$ \\
1.00 & 9.015 & 0.998 & 0.997 & 1.004 & 1.007 \\
& $(1.782)$ & $(1.845)$ & $(1.841)$ & $(1.849)$ & $(1.844)$ \\
\hline
\end{tabular}


TABLE 2. Simulation results for $a_{0}=0.95$ and selected $a_{1}$.

\begin{tabular}{cccccc}
\hline 0.85 & 9.515 & 0.422 & 0.422 & 0.428 & 0.431 \\
& $(1.138)$ & $(1.192)$ & $(1.188)$ & $(1.190)$ & $(1.187)$ \\
0.90 & 9.515 & 0.447 & 0.447 & 0.453 & 0.456 \\
& $(1.138)$ & $(1.178)$ & $(1.173)$ & $(1.175)$ & $(1.174)$ \\
0.95 & 9.515 & 0.472 & 0.472 & 0.478 & 0.481 \\
& $(1.138)$ & $(1.180)$ & $(1.173)$ & $(1.178)$ & $(1.178)$ \\
1.00 & 9.515 & 0.497 & 0.496 & 0.503 & 0.507 \\
& $(1.138)$ & $(1.198)$ & $(1.190)$ & $(1.196)$ & $(1.199)$ \\
\hline
\end{tabular}

TABLE 3. Simulation results for $a_{0}=1.00$ and selected $a_{1}$.

\begin{tabular}{cccccc}
\hline 0.85 & 10.016 & -0.003 & -0.004 & 0.002 & 0.005 \\
& $(1.001)$ & $(1.105)$ & $(1.098)$ & $(1.098)$ & $(1.102)$ \\
0.90 & 10.016 & -0.004 & -0.004 & 0.002 & 0.005 \\
& $(1.001)$ & $(1.074)$ & $(1.066)$ & $(1.067)$ & $(1.073)$ \\
0.95 & 10.016 & -0.004 & -0.004 & 0.002 & 0.005 \\
& $(1.001)$ & $(1.058)$ & $(1.048)$ & $(1.051)$ & $(1.059)$ \\
1.00 & 10.016 & -0.004 & -0.004 & 0.003 & 0.006 \\
& $(1.001)$ & $(1.057)$ & $(1.046)$ & $(1.050)$ & $(1.060)$ \\
\hline
\end{tabular}

\section{REFERENCES}

[1] J. K. Baksalary and A. Markiewicz, Admissible linear estimators in the general Gauss-Markov model, J. Statist. Plann. Inference 19 (1988), 349-359.

[2] J. K. Baksalary, A. Markiewicz and C. R. Rao, Admissible linear estimation in the general Gauss-Markov model with respect to an arbitrary quadratic risk function, J. Statist. Plann. Inference 44 (1995), 341-347.

[3] A. Cohen, All admissible estimates of the mean vector, Ann. Math. Statist. 37 (1966), 458-463.

[4] A. S. Goldberger, Best linear unbiased prediction in the generalized linear regression model, J. Amer. Statist. Assoc. 57 (298) (1962), 369-375.

[5] J. Groß and A. Markiewicz, Characterization of admissible linear estimators in the linear model, Linear Algebra Appl. 388 (2004), 239-248.

[6] D. A. Harville, Extension of the Gauss-Markov theorem to include the estimation of random effects, Ann. Statist. 2 (1976), 384-395.

[7] C. R. Henderson, Estimation of genetic parameters (abstract), Ann. Math. Statist. 21 (1950), 309-310.

[8] C. R. Henderson, Selection index and expected genetic advance, in: Statistical Genetics and Plant Breeding, NAS-NRC 982, Washington, 1963, 141-163.

[9] C. R. Henderson, Best linear unbiased estimation and prediction under a selection model, Biometrics 31 (1975), 423-447.

[10] J. Jiang, A derivation of BLUP-Best linear unbiased predictor, Statist. Probab. Lett. 32 (1997), $321-324$

[11] A. I. Khuri, T. Mathew and B. K. Sinha, Statistical Tests for Mixed Linear Models, Wiley, New York, 1998.

[12] W. Klonecki and S. Zontek, On the structure of admissible linear estimators, J. Multivariate Anal. 24 (1988), 11-30.

[13] L. R. LaMotte, Admissibility in linear estimation, Ann. Statist. 10 (1982), 245-255. 
[14] L. R. LaMotte, Admissibility, unbiasedness, and nonnegativity in the balanced, random, oneway ANOVA model, in: T. Caliński and W. Klonecki (eds.), Linear Statistical Inference (Poznań, 1984), Springer, Berlin, 1985, 184-199.

[15] L. R. LaMotte, On limits of uniquely best linear estimators, Metrika 45 (1997), 197-211.

[16] X. Q. Liu, J. Y. Rong and X. Y. Liu, Best linear unbiased prediction for linear combinations in general mixed linear models, J. Multivariate Anal. 99 (2008), 1503-1517.

[17] T. Mathew, C. R. Rao and B. K. Sinha, Admissible linear estimation in singular linear models, Comm. Statist. Theory Methods 13 (1984), 3033-3045.

[18] C. R. Rao, Estimation of parameters in a linear model, Ann. Statist. 4 (1976), 1023-1037.

[19] C. R. Rao, Estimation in linear models with mixed effects: a unified theory, in: Proc. Second International Tampere Conference in Statistics, Dept. Math. Sci., Univ. of Tampere, 1987, 7398.

[20] H. Sahai and M. M. Ojeda, Analysis of Variance for Random Models. Volume 1: Balanced Data, Theory, Methods, Applications and Data Analysis, Birkhäuser, Boston, MA, 2004.

[21] W. Shiqing, M. Ying and F. Zhijun, Integral expression form of admissible linear estimators of effects in linear mixed models, in: Proc. 2010 International Conference on Computing, Control and Industrial Engineering, IEEE, Wuhan, 2010, 56-60.

[22] C. Stępniak, On admissible estimators in a linear model, Biometrical J. 26 (1984), 815-816.

[23] C. Stępniak, A complete class for linear estimation in a general linear model, Ann. Inst. Statist. Math. A 39 (1987), 563-573.

[24] C. Stępniak, Admissible invariant esimators in a linear model, Kybernetika 50 (2014), 310321.

[25] E. Synówka-Bejenka and S. Zontek, A characterization of admissible linear estimators of fixed and random effects in linear models, Metrika 68 (2008), 157-172.

[26] E. Synówka-Bejenka and S. Zontek, On admissibility of linear estimators in models with finitely generated parameter space, Kybernetika 52 (2016), 724-734.

[27] Y. Tian, A new derivation of BLUPs under random-effects model, Metrika 78 (2015), 905-918.

[28] S. Zontek, On characterization of linear admissible estimators: an extension of a result due to C. R. Rao, J. Multivariate Anal. 23 (1987), 1-12.

[29] S. Zontek, Admissibility of limits of the unique locally best linear estimators with application to variance components models, Probab. Math. Statist. 9 (1988), 29-44.

Ewa Synówka-Bejenka, Stefan Zontek

Faculty of Mathematics, Computer Science

and Econometrics

University of Zielona Góra

ul. prof. Z. Szafrana 4a

65-516 Zielona Góra, Poland

E-mail: e.synowka@wmie.uz.zgora.pl

s.zontek@wmie.uz.zgora.pl

Received 10.10.2017;

revised version 20.12.2018 